\title{
Multimodality Cardiovascular Molecular Imaging Technology
}

\author{
Matthew O'Donnell ${ }^{1}$, Elliot R. McVeigh ${ }^{2}$, H. William Strauss ${ }^{3}$, Atsushi Tanaka ${ }^{4}$, Brett E. Bouma ${ }^{5,6}$, \\ Guillermo J. Tearney ${ }^{5,6}$, Michael A. Guttman ${ }^{7}$, and Ernest V. Garcia ${ }^{8}$ \\ ${ }^{1}$ University of Washington, Seattle, Washington; ${ }^{2}$ Johns Hopkins University School of Medicine, Baltimore, Maryland; ${ }^{3}$ Memorial \\ Sloan-Kettering Cancer Center, New York, New York; ${ }^{4}$ Harvard Medical School, Boston, Massachusetts; ${ }^{5}$ Harvard-MIT Health \\ Sciences and Technology, Cambridge, Massachusetts; ${ }^{6}$ Massachusetts General Hospital, Boston, Massachusetts; ${ }^{7}$ Surgivision Inc., \\ Baltimore, Maryland; and ${ }^{8}$ Emory University, Atlanta, Georgia
}

\begin{abstract}
Cardiovascular molecular imaging is a new discipline that integrates scientific advances in both functional imaging and molecular probes to improve our understanding of the molecular basis of the cardiovascular system. These advances are driven by in vivo imaging of molecular processes in animals, usually small animals, and are rapidly moving toward clinical applications. Molecular imaging has the potential to revolutionize the diagnosis and treatment of cardiovascular disease. The 2 key components of all molecular imaging systems are the molecular contrast agents and the imaging system providing spatial and temporal localization of these agents within the body. They must deliver images with the appropriate sensitivity and specificity to drive clinical applications. As work in molecular contrast agents matures and highly sensitive and specific probes are developed, these systems will provide the imaging technologies required for translation into clinical tools. This is the promise of molecular medicine.
\end{abstract}

Key Words: molecular imaging; cardiovascular; multidisciplinary approach

J Nucl Med 2010; 51:38S-50S

DOI: 10.2967/jnumed.109.068155

\footnotetext{
C
}

ardiovascular molecular imaging is a new discipline that integrates scientific advances in both functional imaging and molecular probes to improve our understanding of the molecular basis of the cardiovascular system. These advances are driven by in vivo imaging of molecular processes in animals, usually small animals. A natural progression of this research is that our increased understanding of these cardiovascular processes will allow us to not only recognize the pathogenesis of the cardiac disease process in individual patients but also translate that knowledge to the development of diagnostic and therapeutic breakthroughs to detect and reverse disease before

Received Feb. 22, 2010; revision accepted Apr. 3, 2010.

For correspondence or reprints contact: Elliot R. McVeigh, Johns Hopkins University School of Medicine, 720 Rutland Ave., 720 Ross

Bldg., Baltimore, MD 21205.

E-mail: emcveigh@jhu.edu

COPYRIGHT ( 2010 by the Society of Nuclear Medicine, Inc. permanent damage takes place. This is the promise of molecular medicine.

To achieve this goal, it is well accepted that a multidisciplinary approach is required, bringing together scientists, engineers, and clinicians. Also, the advantages of each of the available imaging modalities in a multimodality approach is required to understand cardiovascular molecular processes. Advances in cardiovascular molecular imaging using the following 4 modalities are described in this article: ultrasound integrated with other techniques such as photoacoustic imaging; real-time MRI for image-guided therapy; intravascular radiation detectors to measure vascular plaque properties; and optical coherence tomography (OCT) for microscopic coronary plaque imaging. The following sections are excellent representative examples of the progress in and promise of the field of cardiovascular molecular imaging.

\section{NOVEL ULTRASOUND IMAGING APPROACHES}

Ultrasound imaging has been an essential player in cardiovascular diagnostics for more than a generation. However, ultrasound-based tools for molecular imaging have been slow to develop. Molecular imaging studies to date primarily have used microbubbles. They can be coated with polymers or proteins and targeted like other molecular imaging agents (1). Although initially gas-filled, they can also be filled with pharmaceuticals such as chemotherapeutics. In addition to microbubbles, perfluorocarbon-based nanoparticles targeted to cell-bound proteins become highly reflective and are detectable on ultrasound (2).

Because microbubbles are relatively large $(\sim 1 \mu \mathrm{m})$, they are primarily limited to the vasculature. A complementary molecular imaging approach uses a class of biologically targeted agents with significant optical absorption (i.e., not fluorescence) over a limited wavelength range. They are similar in size and binding characteristics to natural macromolecules and can provide high contrast per molecule for photoacoustic imaging. Photoacoustics combines optical and acoustic methods in which contrast is based on optical absorption and spatial resolution scales with ultra- 
sonic frequency. It is not sensitive to the scattering limitations of optical imaging and can provide real-time images at significant image depth with high spatial resolution. Results in animal models have demonstrated its potential for ultrasound molecular imaging beyond the vascular bed, particularly for early diagnosis of common cancers such as those of the prostate (3).

Photoacoustic imaging can sample optical phenomena within tissue to a depth of several centimeters $(4,5)$. Absorbed pulsed laser light in the near-infrared range creates acoustic sources whose strength is proportional to the local absorption at the incident optical wavelength. An image is formed using ultrasound technology, with photoacoustic contrast directly related to optical absorption. Photoacoustics represents one of the most promising techniques for molecular imaging because the optical absorption of bioconjugated nanoparticles can greatly exceed that of tissue over a range of wavelengths in which light can penetrate a few centimeters into the body. Integrating photoacoustic imaging into a real-time ultrasound system can provide simultaneous ultrasound-photoacoustic images. Finally, real-time ultrasound-photoacoustic imaging can be integrated with molecular therapy to produce low-cost tools for molecular medicine.

\section{Recent Experimental Results}

As a specific example of the potential of this technology, we summarize recent results on photoacoustic imaging of nanoparticles targeted to a specific biomarker of vascular inflammation $(6,7)$. Excessive and uncontrolled inflammation plays a key role in atherogenesis. Furthermore, chronic systemic inflammation is a key risk factor for accelerated atherosclerosis. Consequently, the noninvasive identification and quantification of inflammation in the vasculature may provide prognostic information of significant clinical utility. There are several potential molecular biomarkers of endothelial cell inflammation, including cytokines, tumor necrosis factor $\alpha$, and cell adhesion molecules such as intercellular adhesion molecule-1 (ICAM-1). As an initial test of photoacoustic imaging of vascular inflammation, gold nanorods were conjugated to anti-ICAM-1, which binds to the surface of cells overexpressing ICAM-1. Peak absorption of the gold nanorods was tuned to a wavelength of $700 \mathrm{~nm}$ for this study, far from background absorption peaks in tissue.

Of particular interest is a real-time imaging study of a mouse model in which a photochemical reaction to a dye initiates a highly localized inflammation in the inferior vena cava (IVC), as illustrated in Figure 1. Photoacoustic and ultrasound images were acquired before injury using an integrated system and were compared with similar images after injury. In both cases, injections of molecularly targeted agents were synchronized with data acquisition to ensure maximum uptake of particles during photoacoustic imaging. Typical results are presented in Figure 2. Transverse photoacoustic images (Figs. $2 \mathrm{~A}$ and $2 \mathrm{~B}$ ) were taken at

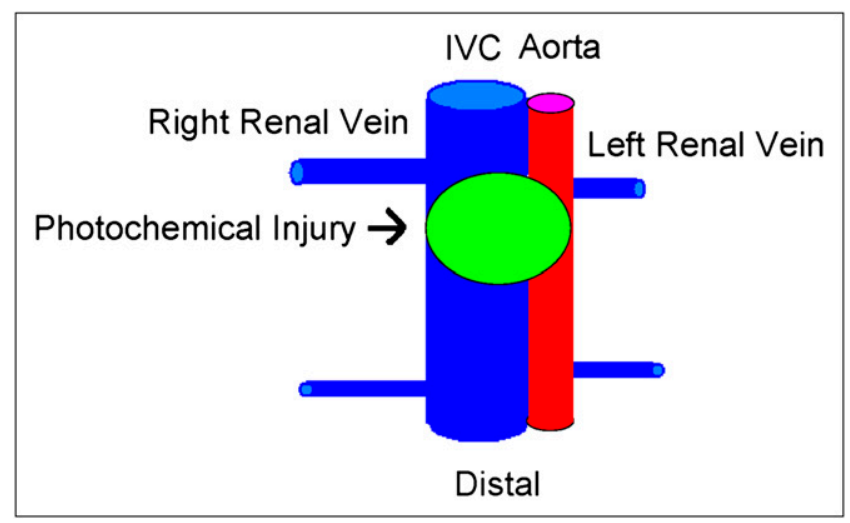

FIGURE 1. Photochemical model of endothelial injury.

the site of injury corresponding to the anatomy shown in Figure 1, along the short axis of the IVC. The photoacoustic intensity after injury (Fig. 2B) was about 10-12 dB higher on average. The longitudinal photoacoustic image (Fig. 2C) was taken at the site of injury along the long axis of the IVC. Photoacoustic intensity at the injury spot (Fig. 2C, dashed circle) is about $10 \mathrm{~dB}$ higher than before injury. The longitudinal view shows that the targeted contrast agent was bound almost exclusively to the injury zone. Clearly, photoacoustic imaging of a targeted molecular contrast agent can identify the primary inflammation site in vivo (7).

To translate these experimental findings into a clinically acceptable molecular imaging tool for interventional procedures, we have also explored integrated, all-optical systems combining photoacoustic and ultrasound imaging in the same device $(8-10)$. Their operating principle is illustrated in Figure 3. A laser pulse at 1 wavelength creates acoustic waves within the device for ultrasound imaging,

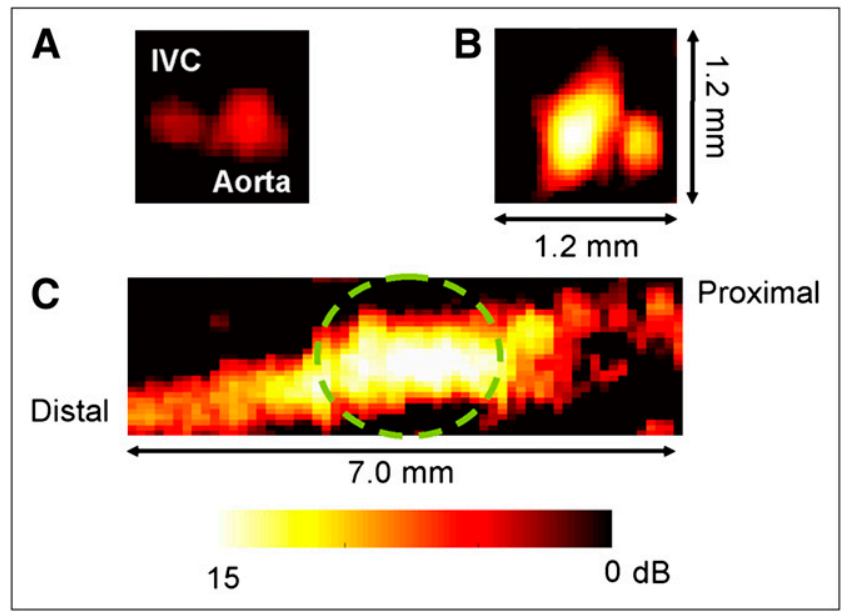

FIGURE 2. (A and B) Transverse photoacoustic images. Intensity after injury (B) is about 10-12 dB higher. (C) Longitudinal photoacoustic image. Intensity at injury spot (dashed circle) is about $10 \mathrm{~dB}$ higher. 
FIGURE 3. All-optical intravascular photoacoustic-ultrasound system in guidewire. PDMS = poly(dimethylsiloxane); ROUT = resonant optical ultrasound transducer.

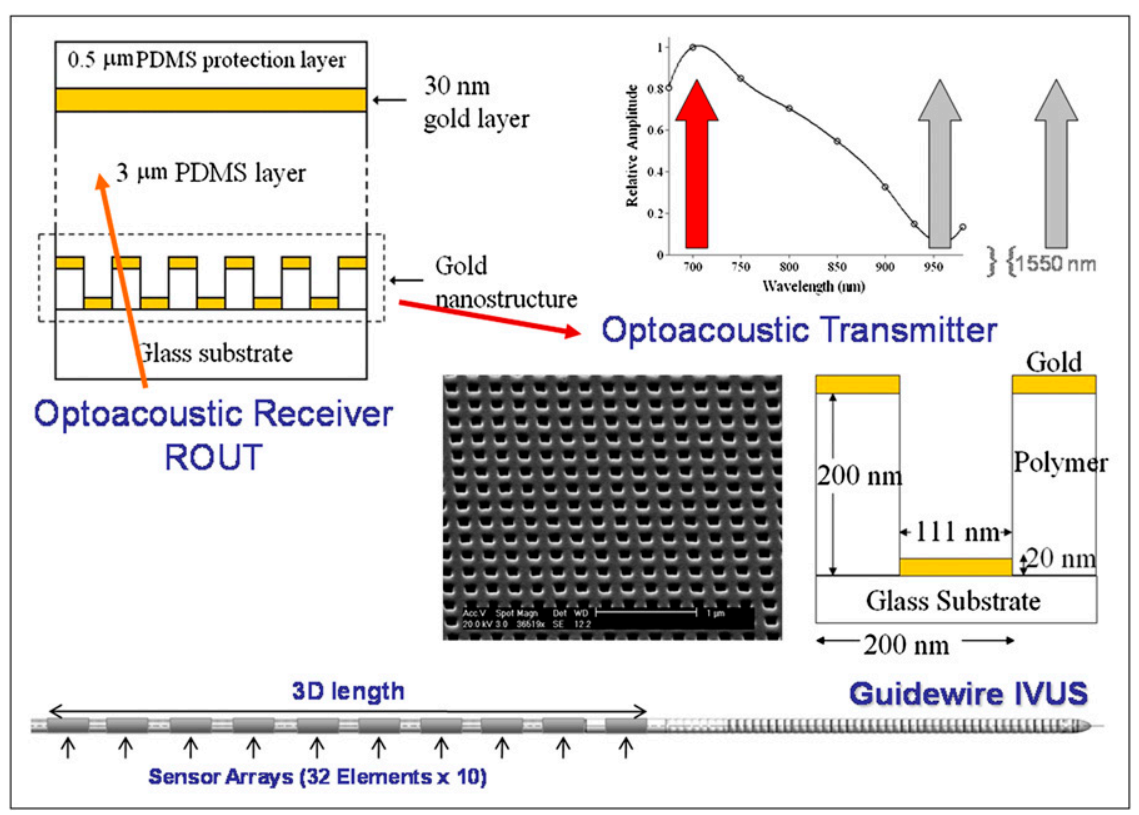

whereas at a different optical wavelength a laser pulse is passed through the device into tissue for photoacoustic imaging. For the specific system presented in this figure, an optical pulse at a wavelength of $700 \mathrm{~nm}$ is efficiently absorbed in a film system (upper left) using a nanopatterned absorber tuned for $700 \mathrm{~nm}$ (lower right) to produce acoustic waves for ultrasound imaging. At a wavelength of around $950 \mathrm{~nm}$, an optical pulse will pass through the device with minimal absorption, depositing most of the optical energy in adjacent tissue for photoacoustic imaging. For molecular imaging with this device, a targeted nanosystem must be tuned for peak absorption at $950 \mathrm{~nm}$ (rather than the 700$\mathrm{nm}$ example). Finally, the structure acts as an optical resonator at $1,550 \mathrm{~nm}$ and can be used as a resonant optical ultrasound transducer (ROUT) to detect acoustic waves when probed with a continuous wave laser at this wavelength. Experimental structures have been constructed demonstrating the potential of this design approach $(9,10)$. Current studies have focused on producing an alloptical intravascular ultrasound (IVUS) guidewire imaging system, as illustrated at the bottom of Figure 3.

\section{Advantages of Integrated Ultrasound-Pulsed Laser System}

Simultaneous ultrasound and photoacoustic images can be produced in real time using either a conventional ultrasound imaging system integrated with a pulsed laser or an all-optical system such as the one presented in Figure 3. Ultrasound images present anatomic information, whereas complementary photoacoustic images present molecular information. If the same detection array is used for both images, then they are automatically coregistered. A common display format for simultaneous ultrasound-photoacoustic imaging is to present regions of high molecular activity in color superimposed on a conventional real-time ultrasound scan presented in gray scale. Images of this type, especially in an IVUS format, coupled with targeted contrast agents for the photoacoustic imaging of molecular events, can bring real-time molecular guidance to interventional cardiology. This is an exciting prospect for the field.

\section{MR IMAGE GUIDANCE AND VISUALIZATION FOR INTERVENTIONAL CARDIAC PROCEDURES}

Over the past decade, several MRI-guided catheter-based applications have been developed. Real-time display of the catheter position on 3-dimensional (3D) MRI is useful for navigating a catheter toward a target tissue. Real-time MRI also offers the physician the ability to monitor the size of the RF lesions through direct imaging of the signal enhancement after the tissue is heated. Other applications include delivery and immediate visualization of intramyocardial injections of stem cells mixed with a contrast agent (11-13) and renal artery stenting (14). Small independent receiver coils can be mounted on devices and used for tracking of devices (15) or creating high-resolution images of tissue just next to the small coils $(16,17)$. The coil locations may be visualized by colorizing images reconstructed from mounted device coil signals and blending them with gray-scale images produced from surface coil signals (18).

Many of the techniques used for intravascular interventions can be applied directly to the guidance of minimally invasive surgical procedures - for example, prosthetic valve placement. The open-chest surgical approach is associated with higher morbidity, but direct access allows superior visualization of anatomy and manipulation of devices for a more durable therapy. With a minimally invasive surgical approach, it may be possible to achieve the best of both surgical and percutaneous approaches: reduce trauma while providing durable therapy. Using interactive real-time MRI, 
the surgeon positioned the prosthetic valve in the correct location at the aortic annulus within $90 \mathrm{~s}$. Ventricular function, blood flow through the valve, and myocardial perfusion were immediately evaluated with MRI $(19,20)$.

\section{Cardiac Interventional MRI System}

New Magnet Configuration. A new 1.5-T magnet design has recently become available; it is a closed-bore design with shorter depth and wider opening (Espree; Siemens Medical Solutions). It has a 120-cm-long bore that is $70 \mathrm{~cm}$ wide; this size provides greater accessibility with only an approximately $20 \%$ loss in imaging speed. The imaging field of view was reduced to $30 \mathrm{~cm}$ but was adequate for many cardiac interventions. This magnet bore is short enough for a surgeon to reach the center of the magnet and wide enough to allow placement and manipulation of instruments over the patient's body.

Interventional Imaging Platform. The platforms developed at the National Heart, Lung and Blood Institute (NHLBI) for interventional real-time MRI (rtMRI) used clinical 1.5-T MRI scanners (Sonata with 8 receiver channels, Espree with 18 channels, and Avanto with 32 channels; Siemens Medical Solutions), with additional software for socket communication over gigabit Ethernet with a Linux workstation (8-central processing unit, 64-bit AMD Opteron; HPC Systems) running custom software for rapid image reconstruction, display, and $3 \mathrm{D}$ rendering $(18,21)$. The workstation is connected directly to the image-reconstruction computer of the MRI scanner for quick access to the raw echo data.

At the beginning of a scan, imaging parameters are sent from the scanner to the workstation for initialization of the reconstruction program. At the end of each image acquisition, a packet of data is sent containing dynamic imaging parameters and the raw MRI data. Commands are sent to the scanner in response to user input via the same network interface.

Real-Time Imaging Features. A useful navigation technique using adaptively oriented projection navigation (18) was designed to facilitate steering of an active device toward target tissue. A device-only projection image and at least 1 standard thin-slice image are displayed together in a $3 \mathrm{D}$ rendering, all updated using real-time imaging. As the user interactively rotates the $3 \mathrm{D}$ rendering, the scanner automatically changes the projection direction, analogous to changing $\mathrm{x}$-ray gantry position during fluoroscopy. This provides a real-time $3 \mathrm{D}$ view of the catheter position and trajectory with respect to the thin-slice image plane. For anatomic context, the thin-slice image is positioned to contain target tissue, and the combination of projection and thin-slice views can be used to navigate the device toward the target.

The NHLBI rtMRI implementation contains the projection imaging features and many other interactive features, which can be controlled by simple keyboard or mouse operation without stopping the scanner. The following is a partial list of interactive features available $(18,22)$ :
- Enable or disable acquisition of selected slices. This feature was often used when slices were initially prescribed for all stages of the procedure, and then only those needed at the time were enabled.

- Display each slice in a separate window and a 3D rendering showing the respective location in space (Figs. 4 and 5). This feature provided simultaneous views of all slices and devices in 1 window, from any angle.

- Highlight device channels in different colors, blended with gray-scale images from surface coils (Fig. 5). The device signal magnitude can be squared to sharpen the device profile.

- Mark reference points. Reference points are displayed as separate graphic objects, such as small colored spheres, in the $3 \mathrm{D}$ rendering. This feature is used to mark anatomic structures for device positioning and targeting (Fig. 5).

- Enable or disable the device-only projection view on selected slices to show the entire device if it exits from the thinner imaging slice.

- Enable or disable adaptive projection navigation mode for 3D projection views of the device.

- Change the acceleration factor.

- Enable subtraction imaging for enhancing contrast injections.

- Enable saving of raw data to files. The same program can be used later to review the images with the same or different options for reconstruction or display. Several display and rendering parameters (such as 3D rendering orientation, highlight colors, and window positions) are also saved. Postprocedure review, therefore, can mimic how the images were displayed during the actual procedure.

\section{Initial Preclinical Procedures}

This cardiac interventional MRI system has been used by the group at NHLBI in several preclinical studies in swine, including endovascular repair of abdominal aortic aneurysms (22), recanalization of chronic total occlusions (23), atrial-septal puncture, and balloon septostomy (24). An ideal application for MRI guidance in the heart is delivery and immediate visualization of intramyocardial injections of stem cells mixed with a contrast agent $(11-15,25)$. Figure 5 shows an example of this type of application. The modified injection catheter is visualized with 2 active coils: the first gives signal along the shaft of the catheter; the second is a small localizer coil at the tip of the catheter. In Figure $5 \mathrm{~A}$ the position of the injection catheter is well visualized in this single frame from a realtime movie of the beating heart. In Figure 5B, a test injection of gadolinium-diethylenetriaminepentaacetic acid contrast agent (diluted 100:1) is easily observed; in this case a saturation pulse was interactively toggled "on" during the injection. In Figure 5C, the injection of $1 \mathrm{~mL}$ of labeled mesenchymal stem cells is observed as a dark 


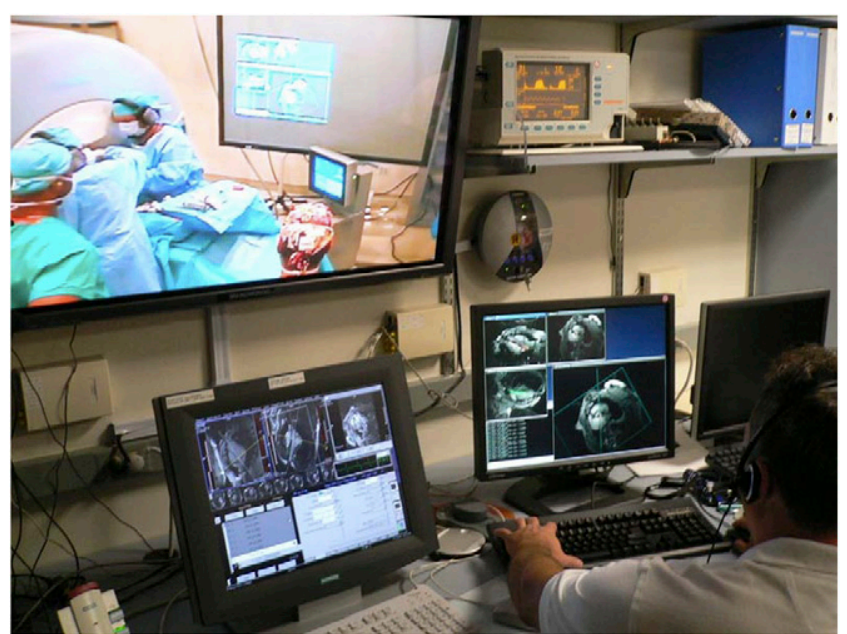

FIGURE 4. Pictures of console room and inside magnet room (seen on LCD screen in front of operator) during swine experiment. Real-time display is directly in front of surgeon. Operator responds to voice commands through voiceactivated microphone to change scan plane views.

region because of the $\mathrm{T} 2 *$ contrast from the intracellular label (13)

All of the images are a superposition of surface coil images with images obtained from a central active guidewire. The guidewire image is reconstructed in green to show the location of the wire (Fig. 6). The prosthetic valve is rotated into position using the signal from the guidewire, which is attached in a fixed location.

The still images in Figure 7 are single frames extracted from the continuous real-time movie that is playing on the screen in front of the surgeon (Fig. 4). Initially, a nitinol guidewire is advanced through the trocar across the native

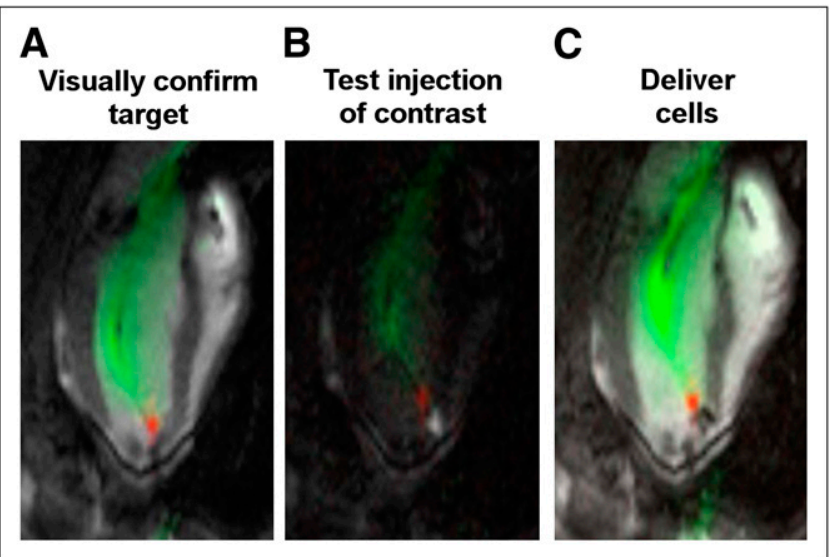

FIGURE 5. Targeting and visualization of intramyocardial injections. Each image is single frame from continuous realtime MRI movie. (A) Injection catheter in position with distal tip against myocardium. (B) Test injection, with interactive saturation applied, shows contrast entering myocardium. (C) Injection of labeled cells; dark signals show presence of cells. aortic valve, and then the prosthetic stent valve on the delivery device is advanced through the apical trocar while its location is observed on the real-time MR image. The prosthetic valve is then advanced on the balloon, along the guidewire, and placed across the native valve in proper position with respect to the coronary artery ostia. The balloon is inflated using $1 \%$ diluted gadopentate dimeglumine (gadolinium-diethylenetriaminepentaacetic acid [Magnavist]; Berlex Inc.) to implant the prosthetic valve under observation with real-time MRI. The balloon is then deflated and removed through the trocar. At this point, the ventricular function resumes, and it is visualized immediately on the real-time MRI.

\section{Advantages of Real-Time MRI for Guiding Cardiac Surgery}

An interactive real-time MRI environment initially developed for use in intravascular procedures (18) has been adapted to guide minimally invasive cardiovascular surgical procedures such as aortic valve placement. Many features were implemented that exploit the advantages of MRI: enhancing visualization of separate antennae or coils mounted on invasive devices, providing multiple oblique slices that are easily adjusted, rendering all slices and landmarks in 3 dimensions, and using accelerated imaging and projection imaging modes to see the entire trajectory of a device receiving signal along its shaft.

Real-time imaging of multiple oblique slices offers many potential advantages. Different views of complicated anatomy may be simultaneously displayed, and individual slices can be interactively turned on or off during a scan as needed. This multiple plane view has been found to be extremely useful for targeting devices. Another potential use is the continuous monitoring of cardiac function in one view during an intervention requiring a different view.

The distinct advantage of MRI for guiding cardiac surgery is the fact that the surgeon can see through the blood, and all of the morphologic landmarks for positioning the device are visible. The short magnet makes it possible to have high-performance real-time MRI available while manipulating the prosthetic valve under image guidance. In addition to aortic valve replacements, other target applications for real-time MRI-guided cardiac surgery are mitral, pulmonary, and tricuspid valve replacements or repairs. Future work will concentrate on the development of novel devices for both surgical and percutaneous access. Also, better-fitting surface coils with optimally placed small coil elements will take advantage of the high number of receivers available on the newer MR imagers.

\section{INTRAVASCULAR RADIATION DETECTORS}

Intravascular sensors can be used to obtain high-resolution information about plaque temperature, lipid content, cap thickness, and inflammation. Specific intravascular devices with thermistors (temperature), plastic scintillators (radia- 


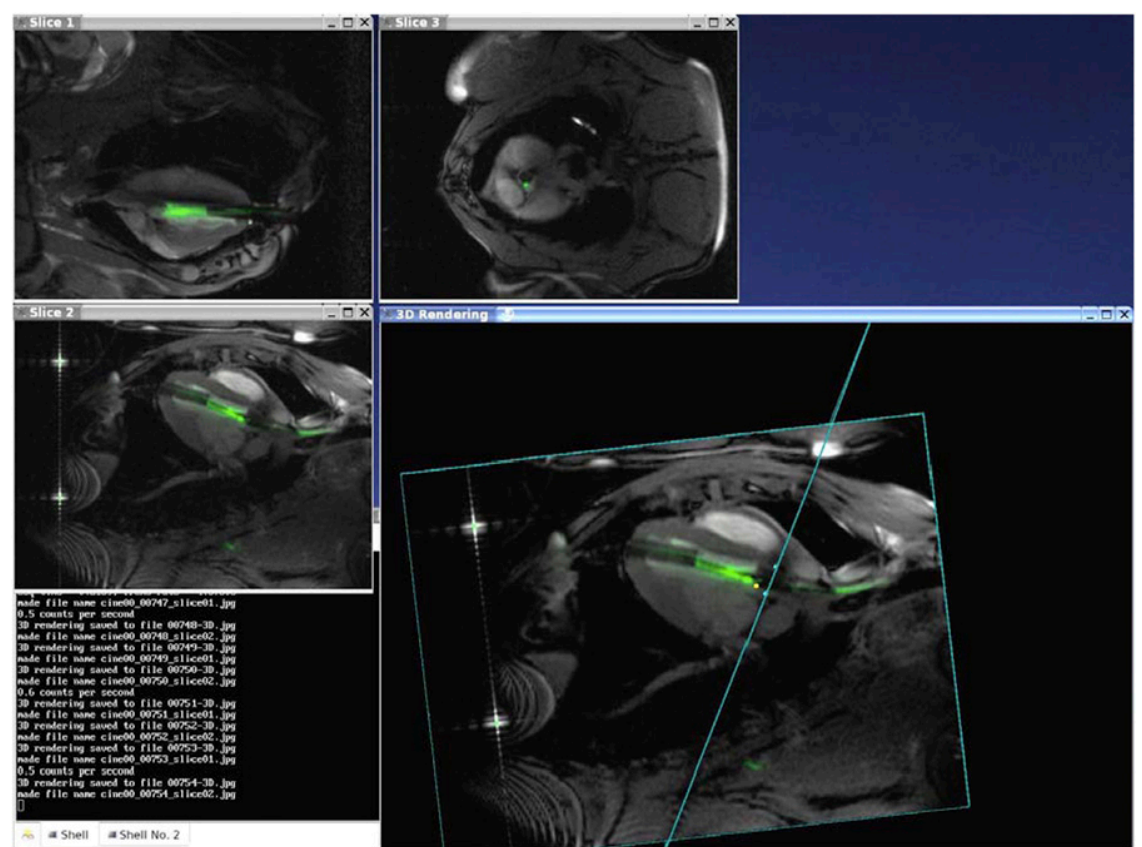

FIGURE 6. Three slices obtained in interleaved fashion showing position of valve delivery device in left ventricle. Top left view shows trochar entering apex of left ventricle; just below that view we can see guidewire (green signal) passing beyond aortic annulus, and in view on top we can see aortic annulus in short-axis orientation, with valve positioned in its center. 3D viewer shows relationship of short-axis slice and anatomic markers on long-axis view. Axial image provided feedback when valve was rotated to align commissures of valve between coronary ostia (cyan dots) before deployment. tion, especially from the positron emission of $\left.{ }^{18} \mathrm{~F}-\mathrm{FDG}\right)$, and optical sensors (for OCT) are used in addition to the well-validated ultrasound detectors. The proximity of intravascular devices to the lesion provides high-spatialresolution information about the atheroma, which will allow a more informed decision about local therapy.

Since the introduction of stress myocardial perfusion 36 y ago (26), studies demonstrate that both single-photon (27) and PET (28) myocardial perfusion studies identify patients at high risk for major acute cardiac events. However, even patients with markedly abnormal PET perfusion scans have only about a $15 \%$ event rate. To increase the specificity of an abnormal perfusion scan, it may be helpful to characterize atheroma in the vessel supplying the ischemic territory. Vulnerable lesions are those with a cap thickness of less than $65 \mu \mathrm{m}$, large lipid lakes, and numerous inflammatory cells. These lesions are more likely to rupture than are lesions with thicker caps, smaller lipid lakes, and fewer inflammatory cells.

Aside from the reduction in lumen diameter, other attributes of atheroma, such as the presence of inflammatory cells (and products they produce, such as matrix
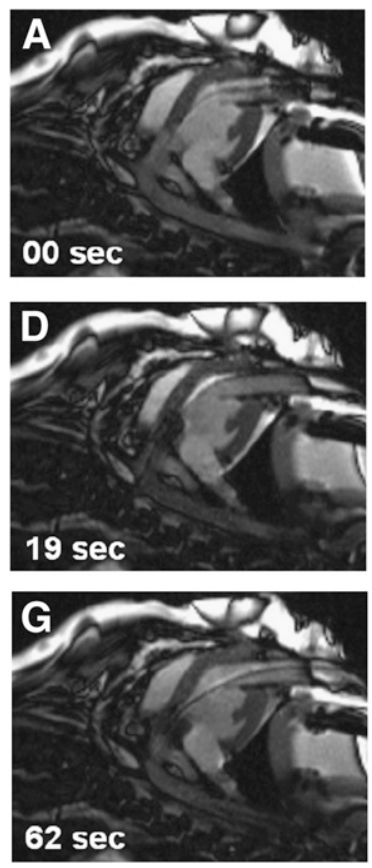
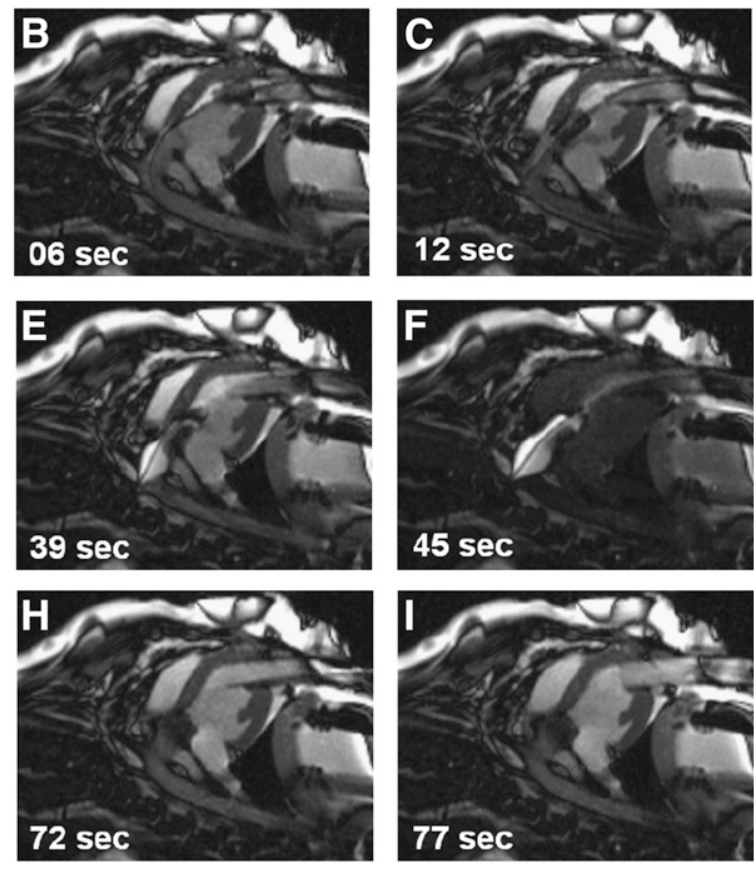

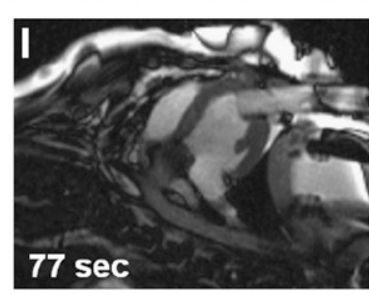

FIGURE 7. Selected frames from realtime MR images displayed within scan room, showing deployment of prosthetic valve. (A) Guidewire is advanced through trocar across native aortic valve. (B) Prosthetic valve is advanced to end of trocar. (C) Prosthetic valve is advanced into position in left ventricular outflow track. (D) Prosthetic valve is inserted across native valve and aligned with coronary ostia and aortic annulus. (E) Balloon filled with dilute gadoliniumdiethylenetriaminepentaacetic acid MRI contrast agent is used to expand prosthetic valve. (F) Interactive saturation is used to enhance visualization of extent of balloon inflation. (G) Balloon is taken down and pulled back through trocar. (H) Guidewire is removed. (I) Delivery device is removed from trochar. Total time of this sequence of pictures is $77 \mathrm{~s}$. (Reprinted with permission of (19).) 
metalloproteinase (29)), may be useful in characterizing the lesion. Numerous other markers-including the presence of low-density lipoprotein, oxidized low-density lipoprotein, apoptosis, cap thickness, total lipid content, and integrin expression-exist.

Histopathologic evidence $(30,31)$ supports the importance of inflammation as a major constituent of vulnerable lesions. One hypothesis is that combining data from a myocardial perfusion scan demonstrating ischemia in a particular territory with specific information on the presence of inflammatory cells in the wall of the vessel perfusing the region will identify lesions at high risk for causing major events.

Inflammatory cells in the atheroma can be identified on the basis of their upregulation of chemotactic receptors (such as monocyte chemoattractant peptide-1 [also called C-C chemokine receptor 2]) (32-34) on the surface of the activated macrophage. An alternative approach uses the known increase in metabolism of the activated cells. Activated macrophages increased their metabolic rate by 10-100 times, compared with baseline. These cells use exogenous glucose to meet their energy needs. As a result, ${ }^{18}$ F-FDG PET scans (in conjunction with CT or MRI for anatomic localization) have been advocated to localize these lesions (35).

Interrogating specific lesions in the catheterization laboratory for the degree of inflammation may distinguish lesions that are likely to progress from those that are stable. This information may be useful in selecting areas for specific treatment, such as balloon angioplasty alone, angioplasty plus bare metal stents, or angioplasty plus drug-eluting stents.

An intravascular radiation-sensitive catheter can localize (and quantify) inflammation in an atheroma. The maximum path length of the positron emitted by ${ }^{18} \mathrm{~F}$ is $3 \mathrm{~mm}$ (mean path, $1 \mathrm{~mm}$ ). This path length is well suited to detection by a 3-French catheter using either a plastic scintillator mated to an optical fiber or a solid-state detector such as cadmium telluride mounted on the guidewire of a coronary artery catheter.

A major concern about the intravascular radiation detector concept is the sensitivity of the device. An analysis of PET/CT images demonstrated that the average ${ }^{18} \mathrm{~F}-\mathrm{FDG}$ uptake in atheroma of the aortic arch (without correction for partial volume) averages approximately $9 \mathrm{kBq} / \mathrm{mL}$ ( $\sim 9,000$ counts/s/mL). Assuming that lesions in the coronaries occupy approximately $0.1 \mathrm{~mL}$ and that only half the positrons originating in the lesion are emitted in the direction of the intravascular detector (based on isotropic emission of radiation), the detector should sense approximately 450 counts/s. We have tested a prototype 1-mmdiameter cylindric plastic scintillator detector $(36,37)$ in ex vivo animal studies with ${ }^{18} \mathrm{~F}-\mathrm{FDG}$ (Fig. 8). The catheter had sufficient sensitivity to detect lesions concentrating one tenth of the anticipated concentration in a coronary atheroma.
A potential problem with the device for the detection of lesions in the coronary arteries is the sensitivity of the device for the 511-keV annihilation $\gamma$-photons originating from tracer deposited in other organs (including the myocardium). Even if the sensitivity for these photons in the low-density (i.e., low-Z) detector material is extremely low (assuming a ratio of $\beta$ - to $\gamma$-sensitivity of 1,000 ), the background radiation from the $\gamma$-radiation will be significant. This problem can be addressed in part by making the detector the shape of a spatula. The $\beta$-particles will deposit their energy, but the $\gamma$-particles will have a high likelihood of traversing the detector with no interaction. This design should raise the ratio of $\beta$ - to $\gamma$-sensitivity to approximately 50,000 , with a marked decrease in background counts.

The feasibility studies demonstrate the sensitivity of a $\beta$-sensitive catheter system. Additional mechanical refinements are needed to optimize the system in anticipation of human studies.

\section{COMPREHENSIVE MICROSCOPIC CORONARY IMAGING FOR VULNERABLE PLAQUES BY OCT}

In the era of drug-eluting stents, the rate of restenosis or target-vessel revascularization has dramatically decreased $(38,39)$. However, the COURAGE trial has shown no significant differences between percutaneous coronary intervention (PCI) and medical-therapy groups in terms of major adverse coronary events, including endpoints of death, myocardial infarction, and hospitalization for acute coronary syndrome (ACS) or myocardial infarction (40). These data have raised the concern that local treatment by PCI as an initial management strategy reduces only ischemia and not major adverse coronary events, beyond the reduction brought about by optimal medical therapy (40). To improve an individual patient's prognosis, we should therefore consider the prevention of ACS that is currently thought to be mainly caused by plaque rupture (41). For this reason, many investigators are pursuing methods to identify coronary plaques at risk for rupture.

Because of the lack of a suitable animal model for atherosclerotic plaque rupture, postmortem study has been the main avenue for advancing our understanding of the pathogenesis of ACS. These autopsy studies have revealed that myocardial infarction results from an acute process of plaque rupture that occurs in modestly stenotic coronary lesions (42). Because invasive coronary angiography projects the vessel lumen, it alone cannot be used to predict plaque rupture. From postmortem studies, plaques containing a large necrotic core with an overlying thin, fibrous cap $(<65 \mu \mathrm{m})$ infiltrated by macrophages, termed thin-cap fibroatheroma (TCFA), are the most common lesions found at the site of plaque rupture (43). Most conventional imaging modalities are unable to identify these lesions because of their low spatial resolution, which prohibits the delineation of thin, fibrous caps. Intracoronary OCT is capable of providing images with less than $10-\mu \mathrm{m}$ resolution and therefore can identify and quantify the thin, fibrous 


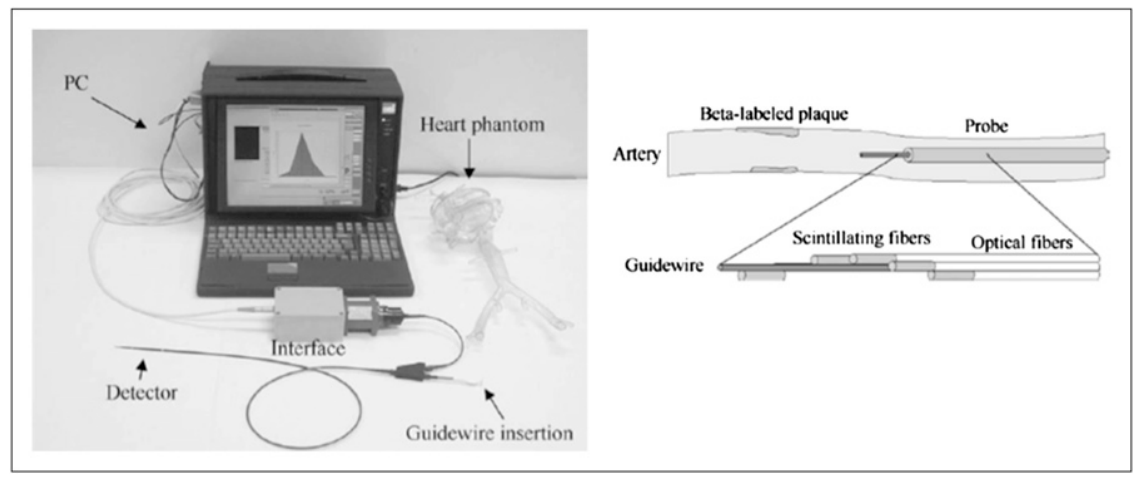

FIGURE 8. ${ }^{18} \mathrm{~F}$ intravascular radiation detector. (Reprinted with permission of (37).)

cap thickness in vivo (44). Moreover, OCT can provide information on the microstructure of the coronary artery and has been shown to be capable of detailed tissue characterization, including characterization of thrombus, supported by histopathologic comparison data (Fig. 9) (45). As such, OCT holds promise not only for detecting vulnerable plaques but also for providing a greater understanding of the pathophysiology of coronary artery disease in patients.

\section{OCT Technology}

Until recently, 2 time domain OCT (TD-OCT) systems have been used for intravascular imaging in the clinical setting: the Massachusetts General Hospital OCT system and the LightLab OCT system (LightLab Imaging). With both TD-OCT systems, an emitted low-coherence light source is divided into 2 paths: a reference path terminating at a reference mirror that moves in a known distance and a sample path to tissue via the coronary catheter. The reflected signals from the sample and the mirror are overlaid and digitized on a photo detector. Only when the lights from both paths have traveled the same optical distance does constructive interference occur. The intensity of interference is translated after processing into an image. For intracoronary OCT, light in the near-infrared range,

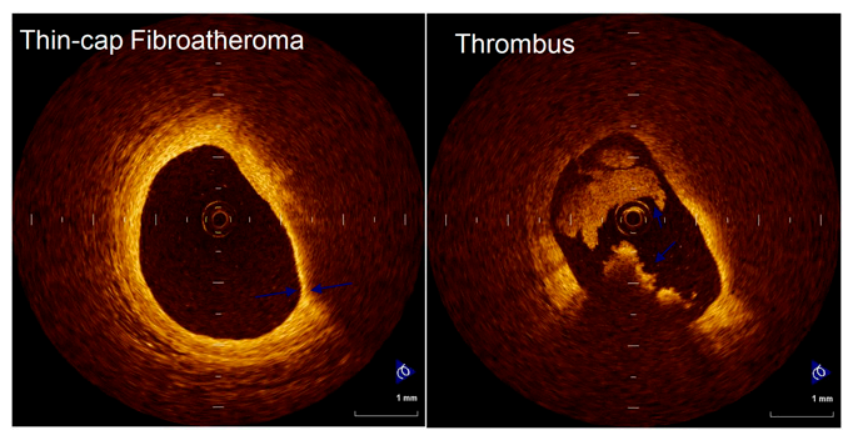

FIGURE 9. Representative cases with TCFA and intracoronary thrombus. (Left) Case of TCFA; thickness of fibrous cap is $50 \mu \mathrm{m}$. (Right) Intracoronary thrombus protruding into vessel lumen from surface of vessel wall. (Reprinted with permission of (51).) with a wavelength centered on around $1,300 \mathrm{~nm}$, is used, providing an axial resolution of approximately $10 \mu \mathrm{m}$ and a penetration depth that ranges between 1 and $2 \mathrm{~mm}$.

The current TD-OCT technology, however, is limited for intracoronary imaging applications. Red blood cells scatter and attenuate the OCT light, making scanning of a long segment impossible in blood-filled vessels. To prevent this signal scattering, a soft balloon occlusion and injection with a transparent flushing medium such as lactated Ringer's solution or radiocontrast have been used to obtain satisfactory coronary images with OCT. Balloon occlusion is difficult to practice in many catheterization laboratories and opens the possibility of inducing undue adverse complications, including damage of the coronary wall and myocardial ischemia.

The second-generation OCT systems-namely frequency domain OCT and optical frequency domain imaging (OFDI)-have been developed to overcome these problems (46).

A narrow-band laser that could rapidly tune its wavelength over a broad spectral width is used for the light source of OFDI. Because the interference pattern is detected as a function of wavelength and used to create images, it is unnecessary to move the reference mirror. When the laser has completed a full spectral sweep, a Fourier transform is applied to the spectral interference pattern to obtain an A-line. This method enables OFDI to provide intracoronary imaging at a much greater rate (100 frame/s) than TD-OCT (47), making it more practical to perform entire-length intracoronary OCT in the clinical setting during a brief, nonocclusive saline or radiocontrast flush (48).

\section{Challenges on Vulnerable Plaques by OCT}

Challenges for Identification of TCFA in Patients with $A C S$. Several clinical TD-OCT studies have reported that TCFAs are more frequently found at the culprit lesions than at the nonculprit lesions in patients with ACS $(49,50)$. Moreover, a recent TD-OCT study clarified that around $70 \%$ of ACS-related plaque rupture seems to be developed from thin, fibrous caps (Fig. 10) (51). These thin caps seem to be disrupted at rest (51). These findings support the 


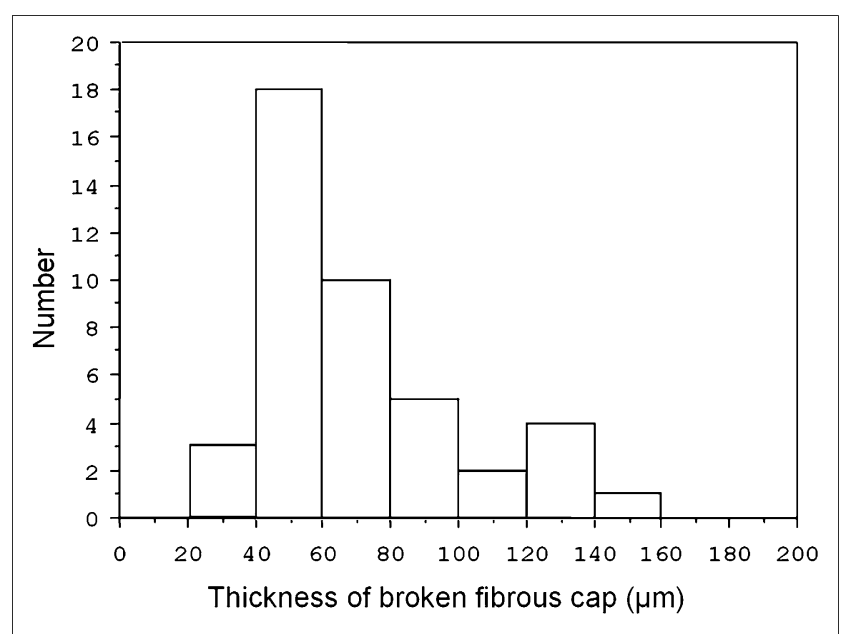

FIGURE 10. Distribution of broken fibrous cap thickness in patients with plaque rupture. Analysis of distribution shows 2 peaks. Around $70 \%$ of patients presented with thickness less than $70 \mu \mathrm{m}$. (Reprinted with permission of (51).)

hypothesis from pathology that cap thickness is one of the most important determinants for plaque disruptions. However, an entire culprit vessel study (52) or a 3-vessel study (53) of TD-OCT also reported that nonruptured TCFAs coexist in the culprit or nonculprit coronary arteries in patients with ACS (Fig. 11). These results raise questions about whether the fibrous cap thickness is the only determinant of plaque rupture in living patients.

Challenges for Macrophage Imaging. Macrophage infiltration is also one of the pathologic features of vulnerable plaques $(43,54-56)$. From several studies of vascular biology, macrophages seem to play key roles in fibrous cap disruption by their potential acts of causing inflammation in plaques (including phagocytosis), secreting proteolytic enzymes such as plasminogen activators and the family of matrix metalloproteinases, or reducing collagen synthesis and enhancing smooth muscle cell apoptosis (57-60). Indeed, previous human IVUS studies demonstrated the relationship between the inflammatory markers, such as high-sensitivity C-reactive protein levels or serum matrix metalloproteinase-9, and the presence of plaque rupture in patients with ACS $(60,61)$. Therefore, macrophages themselves have become one of the challenging targets for coronary imaging. Because of the relatively large size of macrophages $(20-50 \mathrm{~mm})$ and their high degree of optical contrast, investigators have postulated that macrophages may have an OCT signal higher than that of surrounding tissue (62). A study that compared OCT and histology reported the possibility that OCT could identify macrophages or foam cells in individual lesions and potentially enable the quantification of these cells' content by a normalized SD of the OCT signal (Fig. 12) (62).

Translation of OFDI to Clinical Practice. With OFDI, a brief, nonocclusive flush with radiographic contrast can provide a satisfactory OCT image in patients. This ability of OFDI to comprehensively image long coronary segments removes the diagnostic uncertainty that hindered the interpretation of data obtained from previous TDOCT systems (48). A recent report demonstrated that all of the coronary microanatomy and pathology previously visualized by TD-OCT, including lipid pools; calcium; macrophages; thin, fibrous caps; cholesterol crystals; and thrombus, can also be detected by OFDI (48). In addition to native coronary microscopic pathology, stents and stent neointimal hyperplasia were clearly identified in OFDI images from patients (48). This work demonstrates that $3 \mathrm{D}$ microscopic images of long coronary artery segments can now be acquired conveniently in the catheterization lab, and as a result, OFDI may be considered a viable tool for studying the pathophysiology of ACS (Fig. 13).

\section{Future Directions and Clinical Implications}

Natural History of Vulnerable Plaques. Although autopsy studies have contributed to our better understanding of the etiology of ACS, their retrospective nature limits our ability to interrogate a natural history of vulnerable plaques and the clinical significance of these lesions. To know the evolution of vulnerable plaques would improve our understanding of ACS and patients' care.

Currently, only OFDI seems to have the potential to provide reliable microscopic features of vulnerable plaques in whole coronary arteries. OFDI would be a promising device to clarify the natural history of vulnerable plaques. Now, several OCT-OFDI studies have been performed throughout the world.

PCI and Medical Therapy for Vulnerable Plaques: Guided OCT-OFDI. Until recently, IVUS was the gold standard imaging modality to assist in PCI procedures. However, a clinical study reported that OCT for monitoring stent deployments can provide contrast and resolution superior to those of IVUS for depicting arterial disease (63). An OCT study demonstrated different vascular responses between patients with unstable and patients with stable angina (64). Moreover, a recent OCT study with a relatively large cohort showed that semiquantification of lipid content by OCT could predict the development of the no-reflow phenomenon after stent implantation in patients with non-ST-segment-elevation ACS (Fig. 14) (65). These data suggest that better understanding of coronary pathology by OCT may affect PCI, especially for ACS lesions. Clarification is needed of whether OCT-guided PCI could contribute to a better clinical outcome.

A clinical OCT study also showed that statin therapy on TCFA increases the fibrous cap thickness (66). These data suggest that OCT has the potential to assess the efficacy of drug therapy on plaque vulnerability. In either PCI or medical therapy for vulnerable plaques, OCT may play an essential role for the diagnosis and treatment of vulnerable plaques. 


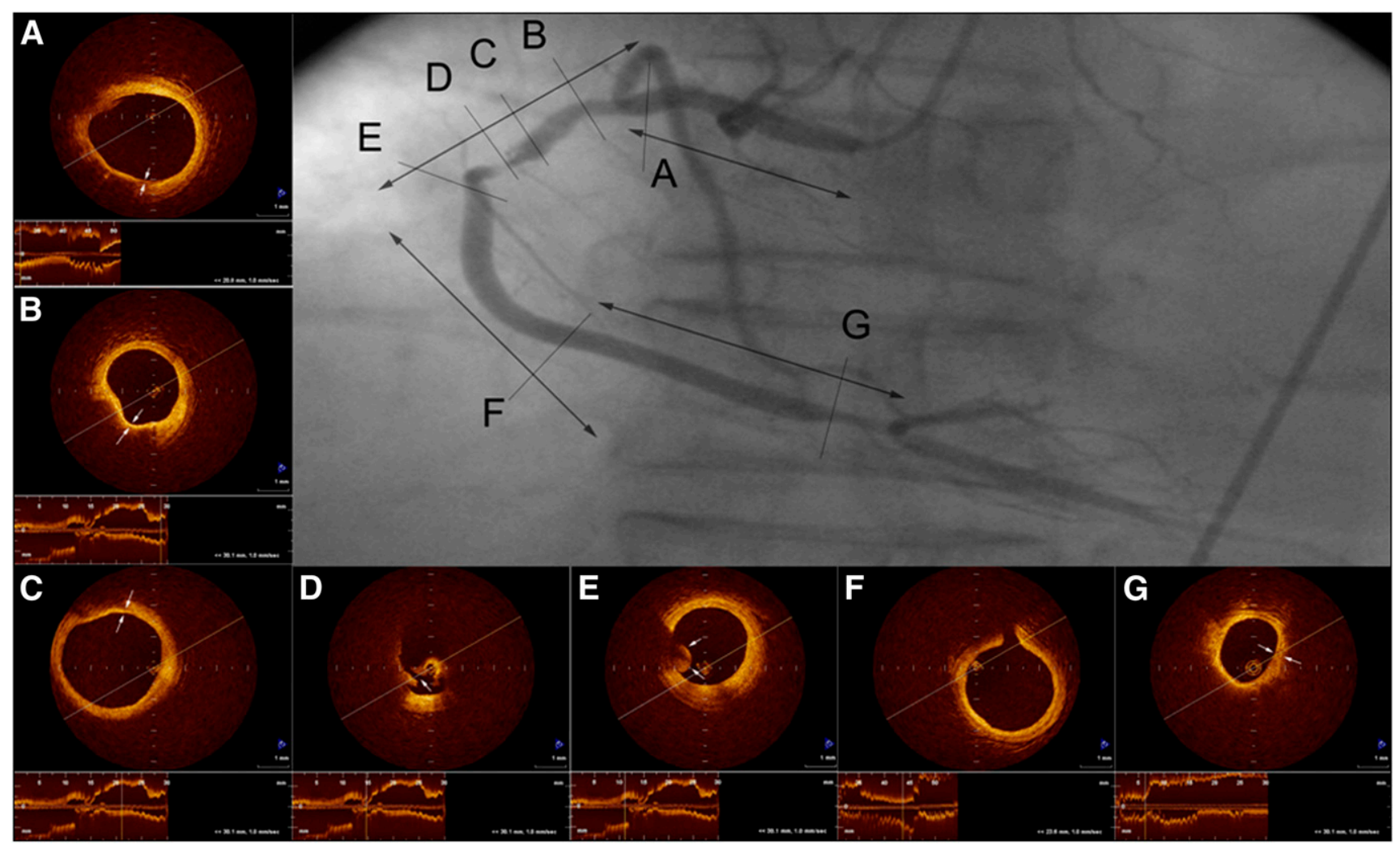

FIGURE 11. OCT, using flush-only technique, images entire culprit coronary artery from distal bifurcation to ostium in ACS patient. Angiogram shows severe lesion in mid portion of right coronary artery and moderate stenosis in distal vessel. (A) Additional TCFA in proximal site; shoulder of fibrous cap is its thinnest part ( $40 \mu \mathrm{m}$, arrows). (B) Proximal TCFA. Center of fibrous cap is cap's thinnest section $(60 \mu \mathrm{m}$, arrows). (C) Proximal end of culprit TCFA. Thinnest point is shoulder of cap (50 $\mu \mathrm{m}$, arrows). (D) Culprit plaque rupture. Cavity formation is clearly visible (arrow). (E) Representative culprit thrombus. OCT clearly shows mass protruding into vessel lumen from surface of vessel wall (arrows). (F) Three layers of arterial wall and branch. (G) Distal lesion. Thick fibrous cap can be observed at $4-0$ 'clock position (240 $\mu \mathrm{m}$, arrows). (Reprinted with permission of (52).)

\section{Advantages of OFDI}

Because OFDI enables the safe and convenient assessment of the 3D microstructure of the coronary wall, including thin, fibrous caps and macrophages, this technol- ogy opens new opportunities for studying vulnerable plaques and determining the predictors of ACS and acute myocardial infarction. Although this field is still in its early stages, OFDI also holds significant promise for prospec-

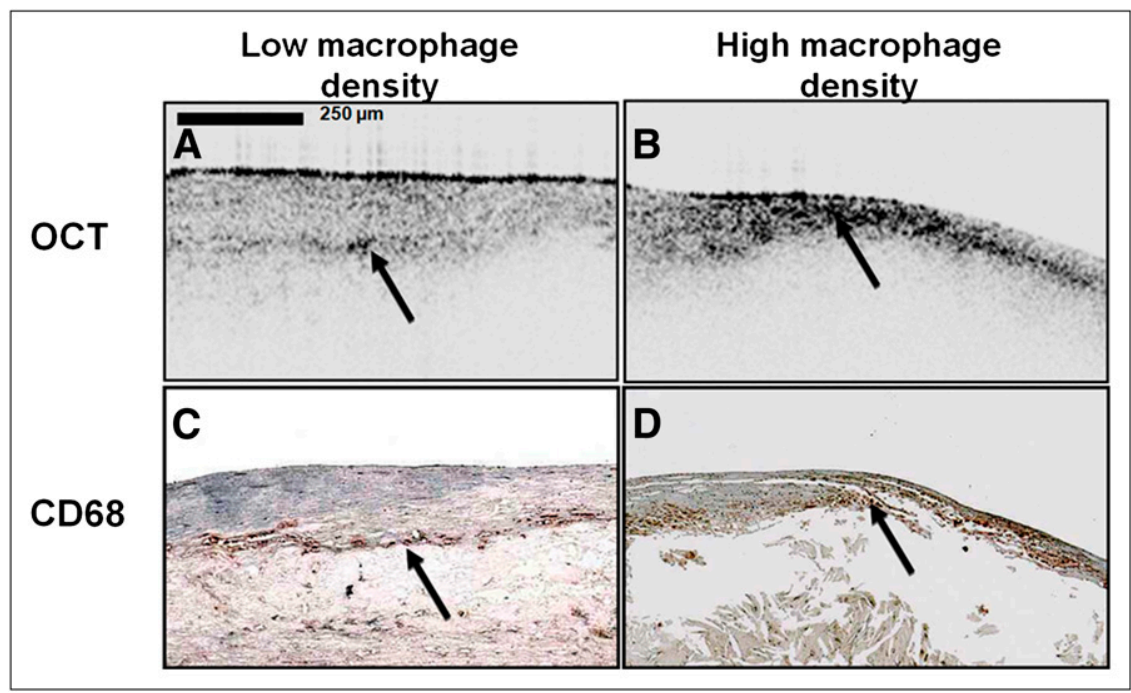

FIGURE 12. (A) Raw OCT images of fibroatheroma with density of macrophages within fibrous cap. (B) Raw OCT images of fibroatheroma with high density of macrophages within fibrous cap. (C) Corresponding histology for A (CD68 immunoperoxidase; $\times 100)$. (D) Corresponding histology for B (CD68 immunoperoxidase; $\times 100$ ). Macrophages (arrows in $\mathrm{C}$ and $\mathrm{D}$ ) could be observed as punctate, highly reflecting regions (arrows in $\mathrm{A}$ and $\mathrm{B}$ ) in raw OCT images. (Reprinted with permission of (62).) 


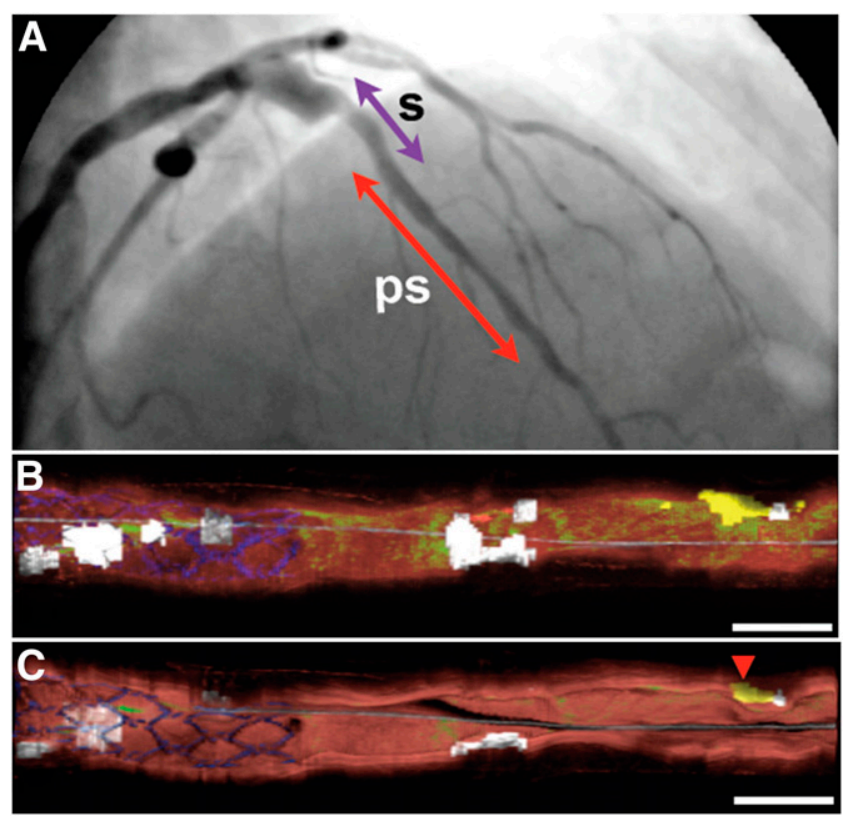

FIGURE 13. OFDI images of left anterior descending artery. (A) Right anterior oblique angiogram after stent deployment, showing stent sites (s) and 4.2-cm OFDI pullback segment (ps). Maximum-intensity projection (B) and cutaway views (C) of 3D volume-rendered OFDI dataset, showing scattered calcium deposits and large lipid-rich lesion at distal portion of ps (arrowhead). Color scale for B and C: red = artery wall; green = macrophages; yellow = lipid pool; blue = stent; white = calcium; gray = guidewire Scale bars in B and C, $5.0 \mathrm{~mm}$. (Reprinted with permission of (48).)

tively identifying high-risk lesions so that they can be treated before the occurrence of an acute coronary event.

\section{CONCLUSION}

Molecular imaging has the potential to revolutionize the diagnosis and treatment of cardiovascular disease. The 2 key components of all molecular imaging systems are the molecular contrast agents and the imaging system providing spatial and temporal localization of these agents within the body. These systems must deliver images with the appropriate sensitivity and specificity to drive clinical applications. In this article, several state-of-the-art imaging technologies for cardiovascular imaging using conventional and intravascular devices have been presented. All are integrated with therapeutic systems and deliver highresolution imaging to drive molecular procedures. Each system-from intravascular arrays for simultaneous ultrasound-photoacoustic imaging, to interventional MRI systems with catheter-mounted devices for imaging and navigation, to intravascular radiation detectors, to intravascular OCT systems - can deliver the required sensitivity and specificity to drive cardiovascular procedures. As work in molecular contrast agents matures and highly sensitive

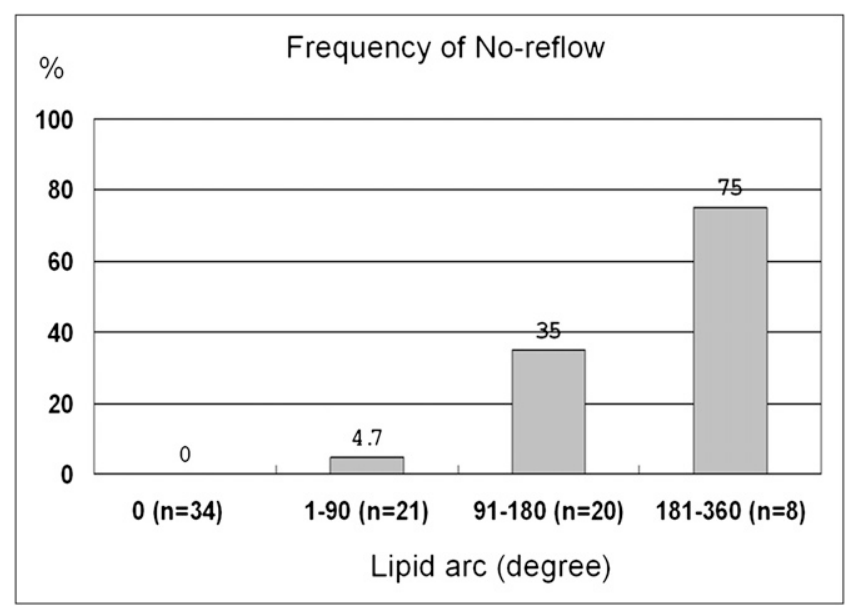

FIGURE 14. Frequency of no-reflow phenomenon and lipid arc. Frequency of no-reflow phenomenon increases according to lipid arc at culprit plaque. Although all patients without lipid plaques (34/34 [100\%]) achieved good reflow, $46 \%$ of patients with lipid-rich plaque (13/28 [46\%]) presented noreflow phenomenon after percutaneous coronary intervention. (Reprinted with permission of (65).)

and specific probes are developed, these systems will provide the imaging technologies required for translation into clinical tools.

\section{ACKNOWLEDGMENTS}

Dr. E.R. McVeigh thanks Joni Taylor, Kathy Lucas, Shawn Kozlov, and Timothy Hunt for animal care and support. Much of the development of rtMRI interventional procedures was a collaboration with the following investigators: Robert Lederman, Keith Horvath, Cenghizhan Ozturk, Parag V. Karmarkar, Amish N. Raval, Venkatesh K. Raman, Alexander J. Dick, Ranil DeSilva, Peter Kellman, and Ming Li. This work was supported in part through the Intramural Research Program of the National Heart Lung and Blood Institute, NIH, DHHS.

\section{REFERENCES}

1. Ellegala DB, Leong-Poi H, Carpenter JE, et al. Imaging tumor angiogenesis with contrast ultrasound and microbubbles targeted to $\alpha_{\mathrm{v}} \beta_{3}$. Circulation. 2003;108: 336-341.

2. Winter PM, Cai K, Caruthers SD, Wickline SA, Lanza GM. Emerging nanomedicine opportunities with perflourocarbon nanoparticles. Expert Rev Med Devices. 2007;4:137-145.

3. Agarwal A, Huang SW, O'Donnell M, et al. Targeted gold nano-rod contrast agent for prostate cancer detection by photoacoustic imaging. J Appl Phys. 2007; 102:064701-1-064701-4.

4. Kruger RA. Photo-acoustic ultrasound: pulse production and detection in $0.5 \%$ Liposyn. Med Phys. 1994;21:1179-1184.

5. Oraevsky AA, Jacques SL, Esenaliev RO, Tittel FK. Time resolved optoacoustic imaging in layered biological tissues. OSA Proc Adv Opt Imaging Photon Migration. 1994;21:161-165.

6. Kim K, Agarwal A, Huang S-W, et al. Photoacoustic imaging of early inflammatory response using gold nanorods. Appl Phys Lett. 2007;90:223901.

7. Kim K, Agarwal A, McDonald AM, et al. In vivo imaging of inflammatory responses by photoacoustics using cell-targeted gold nanorods (GNR) as contrast agent. Proc SPIE. 2008;6856:68560H. 
8. Ashkenazi S, Hou Y, Huang S-W, Buma T, O’Donnell M. High frequency optoacoustic transducers for ultrasonic and photoacoustic imaging and spectroscopy. In: Wang L, ed. Optical Science and Engineering. Oxford, U.K.: CRC Press; 2009:223-238.

9. Hou Y, Kim J-S, Ashkenazi S, Huang SW, Guo LJ, O’Donnell M. Broadband alloptical ultrasound transducers. Appl Phys Lett. 2007;91:073507.

10. Hou Y, Kim J-S, Huang SW, Ashkenazi S, Guo LJ, O'Donnell M. Characterization of a broadband all-optical ultrasound transducer : from optical and acoustical properties to imaging. IEEE Trans Ultrason Ferroelectr Freq Control. 2008;55:1867-1877.

11. Lederman RJ, Guttman MA, Peters DC, et al. Catheter-based endomyocardial injection with real-time magnetic resonance imaging. Circulation. 2002;105: 1282-1284.

12. Kraitchman DL, Heldman AW, Atalar E, et al. In vivo magnetic resonance imaging of mesenchymal stem cells in myocardial infarction. Circulation. 2003; 107:2290-2293.

13. Dick AJ, Guttman MA, Raman VK, et al. Magnetic resonance fluoroscopy allows targeted delivery of mesenchymal stem cells to infarct borders in swine. Circulation. 2003;108:2899-2904.

14. Elgort DR, Hillenbrand CM, Zhang S, et al. Image-guided and -monitored renal artery stenting using only MRI. J Magn Reson Imaging. 2006;23:619-627.

15. Zuehlsdorff $\mathrm{S}$, Umathum R, Volz S, et al. MR coil design for simultaneous tip tracking and curvature delineation of a catheter. Magn Reson Med. 2004;52:214-218.

16. Atalar E, Kraitchman DL, Carkhuff B, et al. Catheter-tracking FOV MR fluoroscopy. Magn Reson Med. 1998;40:865-872.

17. Hillenbrand CM, Elgort DR, Wong EY, et al. Active device tracking and highresolution intravascular MRI using a novel catheter-based, opposed-solenoid phased array coil. Magn Reson Med. 2004;51:668-675.

18. Guttman MA, Ozturk C, Raval AN, et al. Interventional cardiovascular procedures guided by real-time MR imaging: an interactive interface using multiple slices, adaptive projection modes and live 3D renderings. J Magn Reson Imaging. 2007;26:1429-1435.

19. McVeigh ER, Guttman MA, Lederman RJ, et al. Real-time interactive MRIguided cardiac surgery: aortic valve replacement using a direct apical approach. Magn Reson Med. 2006;56:958-964.

20. Horvath KA, Li M, Mazilu D, Guttman MA, McVeigh ER. Real-time magnetic resonance imaging guidance for cardiovascular procedures. Semin Thorac Cardiovasc Surg. 2007;19:330-335.

21. Guttman MA, Lederman RJ, Sorger JM, McVeigh ER. Real-time volume rendered MRI for interventional guidance. J Cardiovasc Magn Reson. 2002;4:431-442.

22. Raman VK, Karmarkar PV, Guttman MA, et al. Real-time magnetic resonanceguided endovascular repair of experimental abdominal aortic aneurysm in swine. J Am Coll Cardiol. 2005;45:2069-2077.

23. Raval AN, Karmarkar PV, Guttman MA, et al. Real-time magnetic resonance imaging-guided endovascular recanalization of chronic total arterial occlusion in a swine model. Circulation. 2006;113:1101-1107.

24. Raval AN, Karmarkar PV, Guttman MA, et al. Real-time MRI guided atrial septal puncture and balloon septostomy in swine. Catheter Cardiovasc Interv. 2006;67:637-643.

25. Hill JM, Dick AJ, Raman VK, et al. Serial cardiac magnetic resonance imaging of injected mesenchymal stem cells. Circulation. 2003;108:1009-1014.

26. Zaret BL, Strauss HW, Martin ND, Wells HP Jr, Flamm MD Jr. Noninvasive regional myocardial perfusion with radioactive potassium: study of patients at rest, with exercise and during angina pectoris. N Engl J Med. 1973;288:809-812.

27. Brown KA, Boucher CA, Okada RD, et al. Prognostic value of exercise thallium201 imaging in patients for evaluation of chest pain. J Am Coll Cardiol. 1983;1: 994-1001.

28. Yoshinaga $\mathrm{K}$, Chow BJ, Williams $\mathrm{K}$, et al. What is the prognostic value of myocardial perfusion imaging using rubidium-82 positron emission tomography? J Am Coll Cardiol. 2006;48:1029-1039.

29. Hartung D, Schäfers M, Fujimoto S, et al. Targeting of matrix metalloproteinase activation for noninvasive detection of vulnerable atherosclerotic lesions. Eur J Nucl Med Mol Imaging. 2007;34(suppl 1):S1-S8.

30. Virmani R, Burke AP, Farb A, Kolodgie FD. Pathology of the vulnerable plaque. J Am Coll Cardiol. 2006;47(8, suppl):C13-C18.

31. Spagnoli LG, Bonanno E, Sangiorgi G, Mauriello A. Role of inflammation in atherosclerosis. J Nucl Med. 2007;48:1800-1815.

32. Ohtsuki K, Hayase M, Akashi K, Kopiwoda S, Strauss HW. Detection of monocyte chemoattractant protein-1 receptor expression in experimental atherosclerotic lesions: an autoradiographic study. Circulation. 2001;104:203-208

33. Blankenberg FG, Tait JF, Blankenberg TA, Post AM, Strauss HW. Imaging macrophages and the apoptosis of granulocytes in a rodent model of subacute and chronic abscesses with radiolabeled monocyte chemotactic peptide- 1 and annexin V. Eur J Nucl Med. 2001;28:1384-1393.
34. Blankenberg FG, Wen P, Dai M, et al. Detection of early atherosclerosis with radiolabeled monocyte chemoattractant protein-1 in prediabetic Zucker rats. Pediatr Radiol. 2001;31:827-835.

35. Rudd JH, Myers KS, Bansilal S, et al. Relationships among regional arterial inflammation, calcification, risk factors, and biomarkers: a prospective fluorodeoxyglucose positron-emission tomography/computed tomography imaging study. Circ Cardiovasc Imaging. 2009;2:107-115.

36. Strauss HW, Mari C, Patt BE, Ghazarossian V. Intravascular radiation detectors for the detection of vulnerable atheroma. J Am Coll Cardiol. 2006;47(8, suppl): C97-C100.

37. Janecek M, Patt BE, Iwanczyk JS, et al. Intravascular probe for detection of vulnerable plaque. Mol Imaging Biol. 2004;6:131-138.

38. Morice MC, Serruys PW, Sousa JE, et al. A randomized comparison of a sirolimus-eluting stent with a standard stent for coronary revascularization. $N$ Engl J Med. 2002;346:1773-1780.

39. Moses JW, Leon MB, Popma JJ, et al. Sirolimus-eluting stents versus standard stents in patients with stenosis in a native coronary artery. $N$ Engl J Med. 2003; 349:1315-1323.

40. Boden WE, O'Rourke RA, Teo KK, et al. Optimal medical therapy with or without PCI for stable coronary disease. N Engl J Med. 2007;356:1503-1516.

41. Fuster V, Badimon L, Cohen M, Ambrose JA, Badimon JJ, Chesebro J. Insights into the pathogenesis of acute ischemic syndromes. Circulation. 1988;77:1213-1220.

42. Falk E. Plaque rupture with severe pre-existing stenosis precipitating coronary thrombosis: characteristics of coronary atherosclerotic plaques underlying fatal occlusive thrombi. Br Heart J. 1983;50:127-134.

43. Virmani R, Kolodgie FD, Burke AP, Farb A, Schwartz SM. Lessons from sudden coronary death: a comprehensive morphological classification scheme for atherosclerotic lesions. Arterioscler Thromb Vasc Biol. 2000;20:1262-1275.

44. Kume T, Akasaka T, Kawamoto T, et al. Measurement of the thickness of the fibrous cap by optical coherence tomography. Am Heart J. 2006;152:755e1-755e4.

45. Tearney GJ, Jang IK, Bouma BE. Optical coherence tomography for imaging the vulnerable plaque. J Biomed Opt. 2006;11:021002.

46. Yun SH, Boudoux C, Tearney GJ, Bouma BE. High-speed wavelength-swept semiconductor laser with a polygon-scanner-based wavelength filter. Opt Lett. 2003;28:1981-1983.

47. de Boer JF, Cense B, Park BH, Pierce MC, Tearney GJ, Bouma BE. Improved signal-to-noise ratio in spectral-domain compared with time-domain optical coherence tomography. Opt Lett. 2003;28:2067-2069.

48. Tearney GJ, Waxman S, Shishkov M, et al. Three-dimensional coronary artery microscopy by intracoronary optical frequency domain imaging. JACC Cardiovasc Imaging. 2008;1:752-761.

49. Jang IK, Tearney GJ, MacNeill B, et al. In vivo characterization of coronary atherosclerotic plaque by use of optical coherence tomography. Circulation. 2005;111:1551-1555.

50. Kubo T, Imanishi T, Takarada S, et al. Assessment of culprit lesion morphology in acute myocardial infarction: ability of optical coherence tomography compared with intravascular ultrasound and coronary angioscopy. J Am Coll Cardiol. 2007;50:933-939.

51. Tanaka A, Imanishi $\mathrm{T}$, Kitabata $\mathrm{H}$, et al. Morphology of exertion-triggered plaque rupture in patients with acute coronary syndrome: an optical coherence tomography study. Circulation. 2008;118:2368-2373.

52. Tanaka A, Imanishi $\mathrm{T}$, Kitabata $\mathrm{H}$, et al. Distribution and frequency of thincapped fibroatheromas and ruptured plaques in the entire culprit coronary artery in patients with acute coronary syndrome as determined by optical coherence tomography. Am J Cardiol. 2008;102:975-979.

53. Fujii K, Masutani M, Okumura T, et al. Frequency and predictor of coronary thin-cap fibroatheroma in patients with acute myocardial infarction and stable angina pectoris a 3-vessel optical coherence tomography study. J Am Coll Cardiol. 2008;52:787-788.

54. Davies MJ, Richardson PD, Woolf N, Katz DR, Mann J. Risk of thrombosis in human atherosclerotic plaques: role of extracellular lipid, macrophage, and smooth muscle cell content. Br Heart J. 1993;69:377-381.

55. van der Wal AC, Becker AE, van der Loos CM, Das PK. Site of intimal rupture or erosion of thrombosed coronary atherosclerotic plaques is characterized by an inflammatory process irrespective of the dominant plaque morphology. Circulation. 1994;89:36-44

56. Libby P. Current concepts of the pathogenesis of the acute coronary syndromes. Circulation. 2001;104:365-372.

57. Moreno PR, Lodder RA, Purushothaman KR, Charash WE, O'Connor WN, Muller JE. Detection of lipid pool, thin fibrous cap, and inflammatory cells in human aortic atherosclerotic plaques by near-infrared spectroscopy. Circulation. 2002;105:923-927.

58. Falk E, Shah PK, Fuster V. Coronary plaque disruption. Circulation. 1995;92: 657-671. 
59. Arbustini E, Grasso M, Diegoli M, et al. Coronary atherosclerotic plaques with and without thrombus in ischemic heart syndromes: a morphologic, immunohistochemical, and biochemical study. Am J Cardiol. 1991;68:36B-50B.

60. Sano T, Tanaka A, Namba M, et al. C-reactive protein and lesion morphology in patients with acute myocardial infarction. Circulation. 2003;108:282285.

61. Fukuda D, Shimada K, Tanaka A, et al. Comparison of levels of serum matrix metalloproteinase-9 in patients with acute myocardial infarction versus unstable angina pectoris versus stable angina pectoris. Am J Cardiol. 2006;97: $175-180$.

62. Tearney GJ, Yabushita H, Houser SL, et al. Quantification of macrophage content in atherosclerotic plaques by optical coherence tomography. Circulation. 2003;107:113-119.
63. Bouma BE, Tearney GJ, Yabushita H, et al. Evaluation of intracoronary stenting by intravascular optical coherence tomography. Heart. 2003;89:317-320.

64. Kubo $\mathrm{T}$, Imanishi $\mathrm{T}$, Kitabata $\mathrm{H}$, et al. Comparison of vascular response after sirolimus-eluting stent implantation between patients with unstable and stable angina pectoris: a serial optical coherence tomography study. JACC Cardiovasc Imaging. 2008;1:475-484.

65. Tanaka A, Imanishi $\mathrm{T}$, Kitabata $\mathrm{H}$, et al. Lipid-rich plaque and myocardial perfusion after successful stenting in patients with non-ST-segment elevation acute coronary syndrome: an optical coherence tomography study. Eur Heart J. 2009;30:1348-1355.

66. Takarada S, Imanishi T, Kubo T, et al. Effect of statin therapy on coronary fibrous-cap thickness in patients with acute coronary syndrome: assessment by optical coherence tomography study. Atherosclerosis. 2009;202:491-497. 